POS $\quad$ PROCEEDINGS

\title{
Dark matter search with the DarkMESA electron beam-dump experiment
}

P. Achenbach, ${ }^{a, b, c, *}$ S. Aulenbacher, ${ }^{a}$ M. Biroth, ${ }^{a}$ S. Caiazza, ${ }^{a}$ M. Christmann,,${ }^{a, b, \dagger}$ A. Denig, ${ }^{a, b, c}$ L. Doria, ${ }^{a}$ J. Geimer, ${ }^{a}$ P. Gülker, ${ }^{a}$ P. Klag, ${ }^{a}$ M. Lauß, ${ }^{a, b}$ M. Littich, ${ }^{a}$ S. Lunkenheimer, ${ }^{a}$ T. Manoussos, ${ }^{a}$ D. Markus, ${ }^{a}$ M. Mauch, ${ }^{a, b}$ H. Merkel, ${ }^{a, c}$ J. Müller, ${ }^{a}$ J. Schlaadt, ${ }^{a}$ B.S. Schlimme, ${ }^{a}$ C. Sfienti, ${ }^{a, c}$ S. Stengel ${ }^{a, b}$ and C. Szyszka ${ }^{a}$

\section{for the MAGIX Collaboration}

${ }^{a}$ Institute of Nuclear Physics, Johannes Gutenberg University Mainz,

Johann-Joachim-Becher-Weg 45, 55128 Mainz, Germany

${ }^{b}$ Helmholtz Institute Mainz, GSI Helmholtzzentrum für Schwerionenforschung, Darmstadt, Johannes Gutenberg University Mainz, 55099 Mainz, Germany

${ }^{c}$ PRISMA+ Cluster of Excellence, Johannes Gutenberg University Mainz, Staudingerweg 9, 55128 Mainz, Germany

E-mail: achenbach@uni-mainz.de

A search for light dark matter (LDM) will be performed by the DarkMESA experiment behind the electron beam-dump in the external beam-line of the MESA accelerator in Mainz, Germany. Various dark sector models motivate the existence of sub-GeV scalar and Majorana or pseudoDirac DM, that are accessible in this type of beam-dump experiments, e.g., by coupling to a dark photon mediator $A^{\prime}$. In the presence of LDM in the dark sector with masses $m_{\chi}<m_{A^{\prime}} / 2$ and a dark coupling strength $\alpha_{D} \sim O(0.1-1)$, the $A^{\prime}$ would predominantly decay invisibly into $\chi \bar{\chi}$ particle pairs. The experiment makes use of the high intensity electron beam available at MESA and will run parasitically to the scheduled program for the external beam mode. The experiment is based on a solid and reliable detection technology and will collect an unprecedented accumulated charge in a few years time, that will extend current exclusion limits for LDM.

\footnotetext{
*** Particles and Nuclei International Conference - PANIC2021 ***

*** 5 - 10 September, $2021 * * *$

$* * *$ Online $* * *$
}

\footnotetext{
* Speaker

${ }^{\dagger}$ Part of doctoral thesis.
} 


\section{The fundamental question}

The mass of baryonic matter accounts for less than $5 \%$ of the mass-energy of the universe. The remaining $95+\%$ is known as dark matter (DM) and dark energy, for which there is clear cosmological evidence. The two disparate forms of matter must have had sufficiently large interactions to have achieved thermal equilibrium during the early phases of the nascent universe. As the Hubble expansion diluted the particle densities, the DM annihilation rate vanished and the co-moving DM density, in relation to baryonic matter, became fixed for all times thereafter. In this thermalequilibrium-origin hypothesis, there must have been a sufficient-high DM annihilation rate to have depleted any excess abundance and thereby concur with current-day observations.

The absence of compelling signals in direct searches for $\mathrm{DM}$ at the $\mathrm{GeV}-\mathrm{TeV}$ mass scale, together with the lack of any evidence of supersymmetry at the Large Hadron Collider, has placed severe constraints on DM models. Recent dark-sector theories predict light DM (LDM) in the $\mathrm{MeV}-\mathrm{GeV}$ mass range [1]. However, the thermal-equilibrium-origin requirement can only be satisfied for LDM if a dark sector contains comparably light new force carriers to mediate the necessary annihilation process. Such mediators must couple to ordinary matter and be neutral under the Standard Model (SM) gauge group. A representative model involves an extra-U(1) gauge boson, dubbed the dark photon $A^{\prime}$ having mass $m_{A^{\prime}}$. The model can be probed by employing highintensity beams from electron accelerators in coordination with detectors dedicated for measuring the interaction of LDM with the SM [2,3].

\section{Exploring the dark sector at MESA}

The Institute of Nuclear Physics in Mainz, Germany, has been operating high-intensity electron accelerators for more than 50 years, most notably the MAinz MIkrotron (MAMI). Searches for hypothetical particles beyond the SM have been performed at MAMI in fixed-target electron-beam experiments [4]. The new Mainz Energy-recovering Superconducting Accelerator (MESA) is going to be situated on the campus of the Johannes Gutenberg University Mainz, next to the existing MAMI accelerator [5]. It will offer about $105 \mathrm{MeV}$ at an $1 \mathrm{~mA}$ (upgradable to $10 \mathrm{~mA}$ ) unpolarized electron beam for fixed, thin target experiments in energy recovery operation (ERL mode) and 150 $-200 \mathrm{MeV}$ at an $150 \mu \mathrm{A}$ polarized electron beam for extracted beam operation (EB mode).

The MAinz Gas Injection target eXperiment (MAGIX) is employing the ERL mode in combination with a gas jet target and two magnetic spectrometers. The very clean and high-intensity beam allows extending the $A^{\prime}$ visible decay searches to lower masses and smaller couplings [6]. Alternatively, a dark photon will decay invisibly if its mass is heavier than twice the mass of the DM candidates. For such a scenario, missing mass experiments with the spectrometer setup are planned, in which an invisibly decaying particle would be identified. A silicon detector within the scattering chamber has been designed, which will allow for the detection of the recoil target protons in coincidence with the scattered beam electron.

Complementary to this approach, the DarkMESA experiment [7] makes use of the highintensity external beam available at MESA, see Fig. 1 for a schematic. It will run parasitically to the scheduled program for the EB mode. The beam-dump in this operation mode will serve as a target, in which feebly interacting particles beyond the SM (for instance axion-like particles or other DM 
candidates) might be produced. Several detectors will be placed 23 meters behind the beam-dump and will be shielded by the walls of the accelerator hall from neutrons or other SM particles that might escape the beam-dump area. The production of $\chi \bar{\chi}$ pairs within a dark photon model as well as the whole experimental setup have been investigated in a detailed simulation.

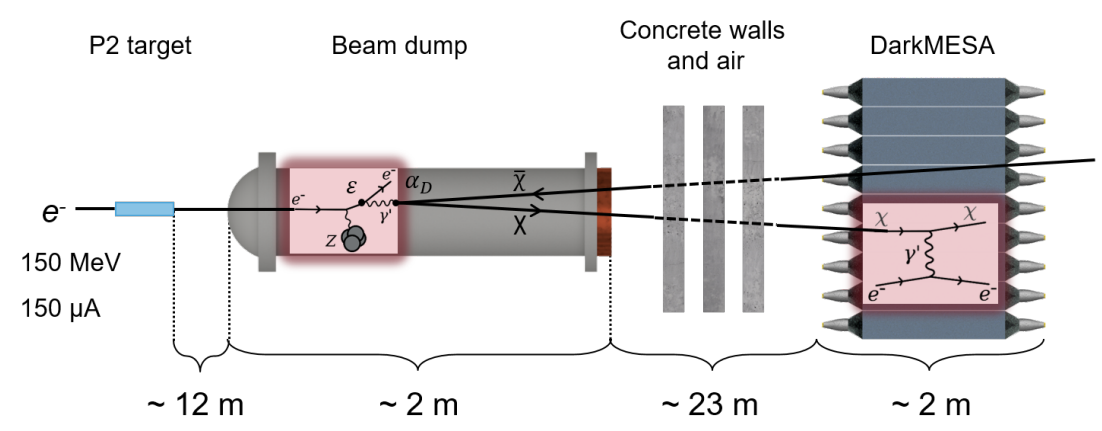

Figure 1: The main elements, from left to right, for the DarkMESA beam-dump experiment (not to scale): The electron beam penetrates a target and impinges upon the dump; Electrons and positrons can undergo dark bremsstrahlung, which can then decay into $\chi \bar{\chi}$ pairs; Shielding will be located before the detectors.

The main detectors will consist of two calorimeters based on Cherenkov light radiators as well as a veto system for rejecting backgrounds. Civil construction for the new experimental hall is expected to be completed in Summer 2022, when installation of the accelerator components and the experimental setup will start. A 25-crystal prototype will then be ready to take data in a Phase A. It will be significantly extended in a Phase B using an established technology based on existing radiators from former experiments. Already in these phases it will be possible to reach the thermal relic targets in an unexplored parameter space of LDM masses. Currently, alternative and more innovative concepts are being investigated, among them a low-pressure, negative-ion, time-projection-chamber and a calorimeter based on radiation shielding window glasses.

\section{Cosmogenic background at the DarkMESA detector site}

The primary issue that the DarkMESA experiment will face is a surfeit of cosmogenic particles passing through the detector site. This plethora, as depicted in Fig. 2 (left), will limit the sensitivity of any detector in measuring rare events, a common problem to all surface detectors. In Fig. 2 (right), the cosmic neutron flux above ground and at the detector site is shown. The passage of the cosmogenic neutrons through the concrete overburden yields an almost double-humped structure. Note a few features: In the high-energy regime, i.e. above $100 \mathrm{MeV}$, the above ground and at-site neutron profiles are nearly identical. This is primarily due to the lack of high $Z$ elements in the concrete. For fast neutrons $(100 \mathrm{keV}$ to $100 \mathrm{MeV})$, the two profiles exhibit the same shape. Only epithermal and thermal neutrons are screened out at the detector site due to their capture by structural elements in the concrete. A boron layered passive shielding can thermalize fast neutrons between $100 \mathrm{keV}$ and $10 \mathrm{MeV}$ without unrealistic overburdens of concrete. 

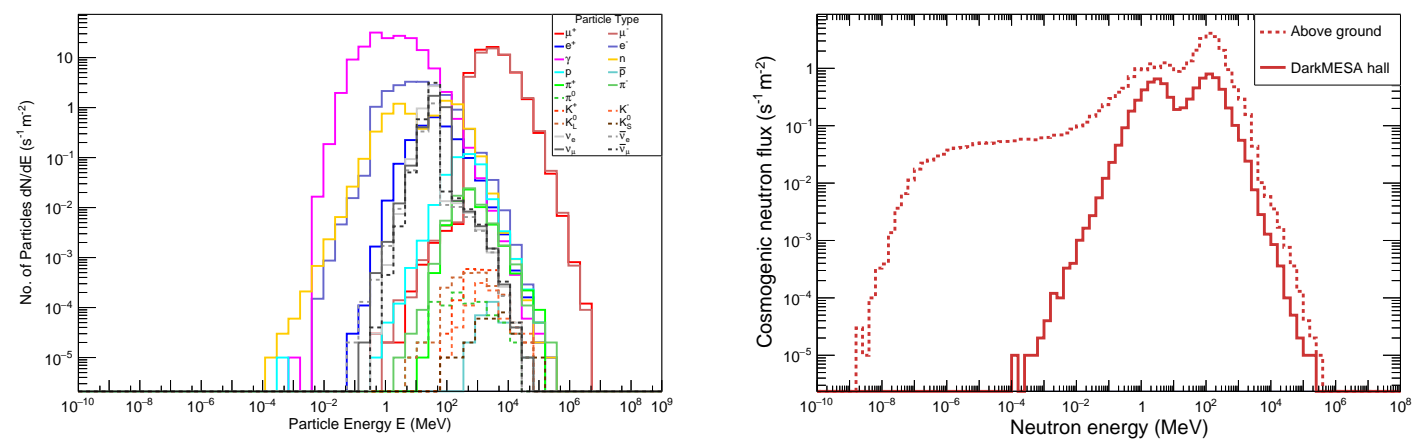

Figure 2: Left: Simulation of the spectrum of all components of cosmogenic particles penetrating the 1-m concrete overburden into the DarkMESA experimental detector site. Right: Simulation of the cosmogenic neutron flux above ground (dotted line), and underground at the detector site (solid line).

\section{Conclusions}

Within the context of dark-sector theories, LDM is an attractive candidate for the DM making up the vast bulk of the mass of our universe. The DarkMESA experiment is employing calorimetric detectors to search for LDM. The detectors will be situated behind the beam-dump of the highintensity beam of electrons from the MESA accelerator. The discovery potential of DarkMESA includes conjectured $\chi$ particles, with a mass lower than half a dark photon mass. The sensitivity includes regions of parameter space wherein the LDM is predicted to have the required relic density consistent with the anticipated DM density that has been observed indirectly in cosmology. The sensitivity of the setup to other DM scenarios such as axion-like particles is under study.

\section{Acknowledgments}

This work was supported in part by the PRISMA+ Cluster of Excellence "Precision Physics, Fundamental Interactions and Structure of Matter" and by the Helmholtz-Gemeinschaft Deutscher Forschungszentren (HGF) with a HGF-Exzellenznetzwerk.

\section{References}

[1] Feebly-Interacting Particles: FIPs 2020 Workshop Report, 2021. arXiv:2102.12143.

[2] J. D. Bjorken et al., Phys. Rev. D 80 (2009) 075018.

[3] Working Group Report: New Light Weakly Coupled Particles, 2013. arXiv: 1311.0029.

[4] A1 Collaboration, H. Merkel et al., Phys. Rev. Lett. 112 (2014) 221802.

[5] K. Aulenbacher, AIP Conf. Proc. 1563, p. 5, 2013.

[6] L. Doria et al., PoS 360, p. 22, 2021.

[7] M. Christmann et al., Nucl. Instrum. Methods Phys. Res. A 958 (2019) 162398. 